\title{
Human Oscicular Chain Articulations: Asymmetric Sound Transmission
}

\author{
Articulaciones de la Cadena Oscicular Humana: Transmisión Asimétrica del Sonido
}

\author{
"Luis Miguel Ramirez Aristeguieta \& *"Luis Ernesto Ballesteros Acuña
}

RAMIREZ, L. M. \& BALLESTEROS, L. E. Human oscicular chain articulations: Asymmetric sound transmission. Int. J. Morphol., 28(4):1059-1068, 2010.

SUMMARY: The mechanism for conducting acoustic energy via middle ear oscicles is still a controversial topic and will remain so until consensus is reached regarding whether it amplifies or reduces sound transmission. 22 paired human temporal bone blocks and 1 left block were studied. Digital measurements were taken of the tympanic membrane, oscicular articulations and the oval window; the results were then correlated. A relationship of non-lineal areas was found amongst the structures being studied, suggesting a sound transmission relationship combining both sound reduction and amplification. A complex relationship of levers could be observed originating in an oscicular and articular asymmetric relationship suggesting an amplifying function in initial sound transmission and also a final reducer destination for such conduction.

KEY WORDS: Malleus; Incus; Stapes; Tympanum; Incudomalleolar joint; Incudostapedial joint; Oval window; Stapedial muscle.

\section{INTRODUCTION}

The peripheral filtrate in sound energy conduction in the middle ear hypothesis must take the variables implicated in its conception into account (Coleman \& Colbert, 2010). This theory affirms that understanding variables such as mass, toughness, elastic resistance and the frictional resistance of oscillating bodies (in this case, the oscicles) is fundamental in configuring the impedance of sound transmission through air, understanding impedance to be resistance caused by the opposition of each verbratile element in conducting sound through air to a liquid environment. Even though the tympanic membrane and the small bones generate an adjustment in impedance of sound transmission through air and liquid during hearing, the transmission-modulation mechanism acting through oscicular configuration with asymmetric levers is still a controversial topic since it still has not been defined whether they amplify, reduce or harmonisingly amplify and reduce sound transmission.

A clear idea of the anatomy of the middle ear (externally limited by the tympanic membrane and internally in the oval window) leads to understanding the conductive component in sound transmission. The tympanic membrane separates the external ear's tympanic cavity; it is semitransparent, thin and is oval-shaped, having a diameter of around $8-10 \mathrm{~mm}$. It is thinner above than below and forms a fifty-five degree angle with the floor of the external ear conduct. The annular boundary surrounds its whole perimeter to become inserted into the tympanic sulcus (Kassem et al., 2010). This consists of a flaccid superior membrane (limited by the anterior and posterior tympanum-malleolar ligaments) and a tense, inferior membrane representing most of the tympanic membrane. The malleus handle is firmly bound to its internal facet as far as its centre or navel which is inflexed towards the tympanic cavity, making this a flat cone which is convex inside. The incus is bound to the malleus and, in turn, the stapes to the latter through incudomalleolar and incudostapedial synovial joints, respectively. All are involved in transmitting sound from an air environment to a liquid one and through each small bone's respective and corresponding (complementary) articular area.

\footnotetext{
${ }^{*}$ Professor and Director of the Stomatognathic System and Morphophysiology Group, Universidad Santo Tomas de Aquino, Bucaramanga, Colombia.

${ }^{*}$ Full Professor Basic Science Department, Universidad Industrial de Santander, Bucaramanga, Colombia.
} 
The small bones' morphology is not symmetrical, having particular characteristics making them into levers when they become combined. The malleus has a large, ovoid head, a handle or manubrium which is attached to the tympanic membrane and a thin neck containing an anterior and a lateral apophysis. The articular facet of this bone is located in the head. The incus seems to be more a flattened premolar tooth, having two divergent roots which are the superior or short apophysis and the inferior or long one. It has two facets, an anterior one for the malleus in the shape of a saddle and an inferior or lenticular one for the stapes which is perpendicularly located to the axis of the long apophysis of the incus which, in turn, is located parallel and medial to the malleus handle and the tympanic membrane.

The stapes has a head, anterior and posterior arms or apophysis (cruras) and a base or footplate which is fixed with the annular ligament to the oval window of the internal ear. The head, contrary to the footplate, has a lateral and perpendicular direction to the tympanic membrane, malleus handle and long apophysis of the incus. The head of the stapes contains a facet which articulates with the lenticular apophysis of the incus. The anterior apophysis curves from the head of the stapes towards its footplate in a medial direction, as does the posterior apophysis; the anterior apophysis is less curved and shorter than the posterior apophysis (Olsewski, 1989; Olsewski, 1990; Guyton \& Hall, 2007; Asai et al., 1999).

Even though the structure (dimensions, weight) and function of the small bones and the tympanic membrane has been studied in humans (foetuses, children and adults), understanding their amplifying dynamics continues being a controversial topic (Olsewski, 1989; Olsewski, 1990; Anson \& Donaldson, 1991; Nakajima et al., 2005). The patterns of transmitting forces and their mechanics have also been studied with mathematical models for reconstructing the particular forms of the small bones so that they can then be processed using finite element model studies (Tonndorf \& Khanna, 1972; Sun et al., 2002; Arensburg \& Nathan, 1971; Serrat et al., 1988; Aycan et al., 1990). There seems to have been common interest in the morphometry of the ossicle chain in the human middle ear for surgical purposes and also in designing and constructing electromagnetic implants, using precise measurements and thereby managing to reproduce the transmission of sound energy (Asai et al.; Whyte et al., 2003; Proop et al., 1984; Wassif, 1951; Farahani \& Nooranipour, 2008; Wengen et al., 1995; Murugasu et al., 2005; Nishihara \& Goode, 1994; Mills, 1991).

Interestingly, Crevecoeur (2007) has shown how the small bones are morphometrically stable in both ethnically and geographically different pre-historic and historic human populations, also pointing out that the size of the cranium and the body have no correlation with that of the oscicles, thereby having substantial phylogenetic value. This would mean that there are very few anatomical variations for these osseous structures.

Such small bone composition or oscicular mechanism makes movement through the air (low density) in analogous movements having greater density in the cochlear liquid. The malleus handle reproduces tympanic membrane movements whilst the malleus and the incus rotate together around an axis running from the short apophysis and the posterior ligament of the incus to the anterior ligament of the malleus. Thus, when the membrane and the handle move in a medial direction, the long apophysis of the incus moves in the same direction pushing the base of the stapes towards the labyrinth and the perilympha with a piston-type, irregular rotational movement (Heiland et al., 1999; Hato et al., 2003). The force per surface unit of oscillating area increases 20-22 times at the base of the stapes compared to that of the tympanic membrane since there is 15-17 times more area in the tympanic membrane than in the oval window (Fig. 1). If the oscicular chain were not present, then more than $90 \%$ of sound energy would not be transmitted and, if it is taken into account that only $60 \%$ of sound is normally transmittable, then the importance of the oscicular chain in such conduction may be deduced (Olsewski, 1989; Olsewski, 1990; Guyton \& Hall; Asai et al.).

This article was designed to show the mechanical and conductive transmission relationship of the articular areas formed by incudomalleolar and incudostapedial joints and the measurements in each oscicle and in the area of the

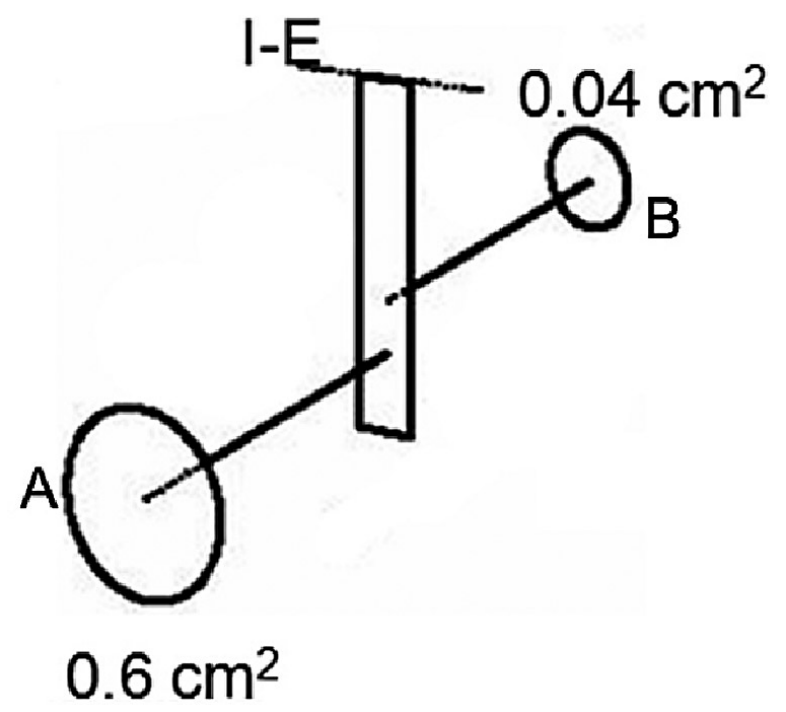

Fig. 1. The relationship of areas $\left(\mathrm{cm}^{2}\right)$ between the tympanic membrane (A) and the oval window (B). I-E, incudostapedial joint. 
tympanic membrane and the stapes footplate in the oval window. The idea was to analyse the influence of each area individually and then taken together so as to clarify their dynamics.

\section{MATERIAL AND METHOD}

Twenty-three temporal blocks without lesion $(7 \mathrm{~cm}$ each side) were extracted from 11 males and 1 female who had not been claimed from the Forensic Medicine Institute in Bucaramanga, Colombia. Twenty-one adult specimens were paired and just one sample was taken from the left-hand side. The samples were frozen and then immersed in $0.9 \%$ saline solution with timerozal $(1: 10,000)$ for one week (to keep the tissue soft) until use for dissection purposes. Formaldehyde was not used to avoid fixing the tissue, thereby hampering dissection of the blocks.

The temporal blocks were cut with $15 x$ magnification, manual instrumentation and micro-motor plus different sized round drill bits. Two access points were used: one by the medial cranial fossa to gain access to the oscicles and another by the external acoustic meatus to gain access to the tympanic membrane. The first cut was aimed at gaining access to the middle ear via the epitympanic recess so as to extract the oscicles. They were cleaned with oxygenated water for 3 days and then their articular areas were measured with a Mitutoyo electronic calibrator. The following areas were measured: the area of incudomalleolar joint in the malleus, the area of incudomalleolar joint in the incus, the area of lenticular articulation in the incus, the area of lenticular articulation in the stapes and the area of articulation of the footplate of the stapes in the oval window.

The different areas were then calculated from the recorded measurements, using the ellipse formula (expressed in $\mathrm{mm}^{2}$ ) due to each surface's closed curves and measurements on the semimajor and semiminor axes. Side differences were then compared (right- and left-hand sides of the cadaver) for measuring the articular areas of the oscicles from the incudomalleolar joint to the oval window, seeking to find such differences in the paired samples.

The second approach consisted of widening the external acoustic meatus as far as the tympanic membrane for taking in situ measurements of it and so as not to lose its spatial disposition and natural tension, keeping its morphology unaltered. Two records of length (semimajor axis of the ellipse) and breadth (semiminor axis of the ellipse) were taken, the first on the axis of the handle and the second on a perpendicular axis to the first (Fig. 1).
Each measurement was recorded in data collection format. Parametric tests were made, as was a Pearson correlation matrix for the articular areas. The investigation was approved by the University's Ethics Committee.

\section{RESULTS}

Measurements were obtained for the tympanic membrane (Fig. 2) on two axes. An average $8.79 \mathrm{~mm}$ (SD 0.73 ) length was found on the axis of the handle and average transverse length to the former measured $6.88 \mathrm{~mm}$ (SD 0.82).

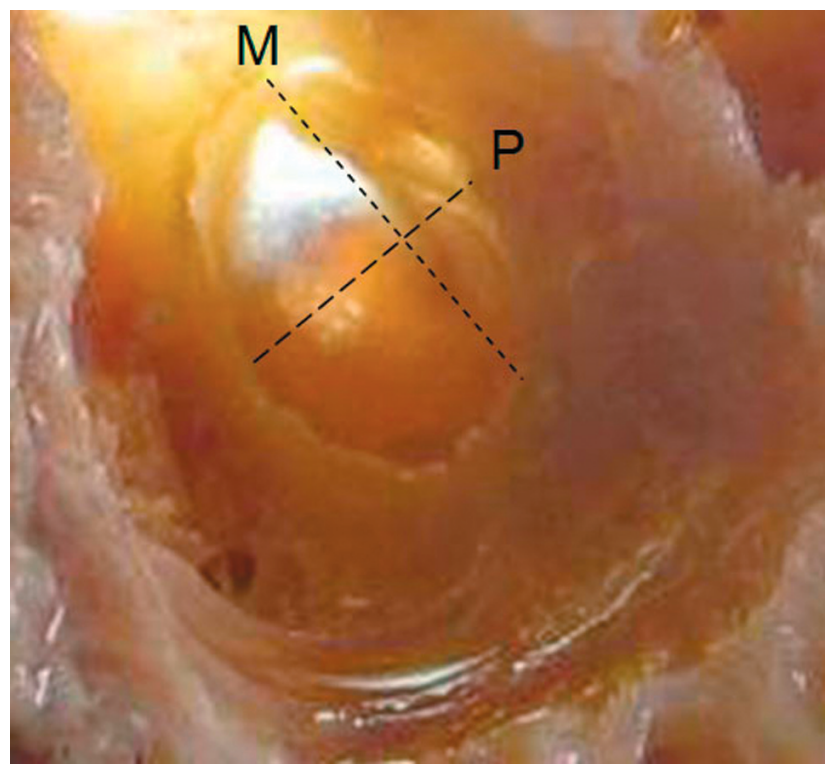

Fig. 2. Tympanic area length on the handle axis (M) and medio-lateral axis perpendicular to that of the handle $(\mathrm{P})$.

Three measurements were taken for the articular facet of the malleus (Fig. 3) on its semiminor axis and one on its semimajor axis. Average lateral measurement on the semiminor axis was $1.86 \mathrm{~mm}(0.29 \mathrm{SD}), 1.56 \mathrm{~mm}(0.26 \mathrm{SD})$ intermediate and $1.68 \mathrm{~mm}(0.2 \mathrm{SD})$ medial. A $2.6 \mathrm{~mm}$ length $(0.3 \mathrm{SD})$ was observed on the semimajor axis (superior-inferior).

Three measurements were also taken on the articular facet (malleolar) of the incus along the semiminor axis (medio-lateral) and one on the semimajor axis (antero-posterior). Averages on the most posterior semiminor axis were $1.42 \mathrm{~mm}$ $(0.27 \mathrm{SD}), 1.45 \mathrm{~mm}(0.2 \mathrm{SD})$ on the intermediate and 1.82 $\mathrm{mm}(0.14 \mathrm{SD})$ on the most anterior. The measurement was $2.93 \mathrm{~mm}(0.19 \mathrm{SD})$ on the semimajor axis.

An average $0.81 \mathrm{~mm}(0.1 \mathrm{SD})$ measurement was obtained on the semimajor axis (antero-posterior) on the articular facet (stapedial) of the incus (Fig. 4) and $0.56 \mathrm{~mm}(0.09$ $\mathrm{SD})$ on the semiminor superior-inferior axis. 

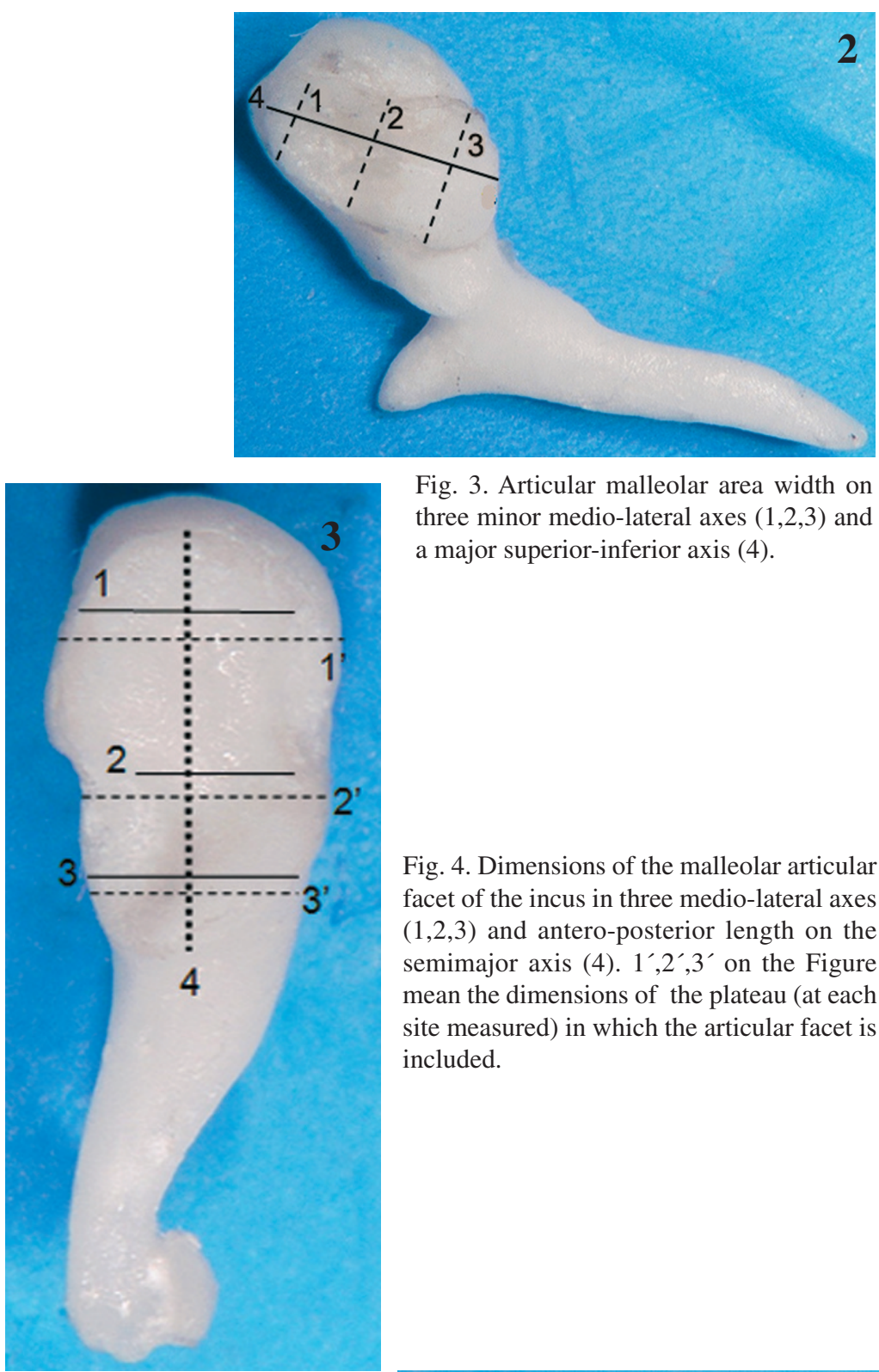

Fig. 3. Articular malleolar area width on three minor medio-lateral axes $(1,2,3)$ and a major superior-inferior axis (4).

Fig. 4. Dimensions of the malleolar articular facet of the incus in three medio-lateral axes $(1,2,3)$ and antero-posterior length on the semimajor axis (4). 1', 2', $3^{\prime}$ on the Figure mean the dimensions of the plateau (at each site measured) in which the articular facet is included.

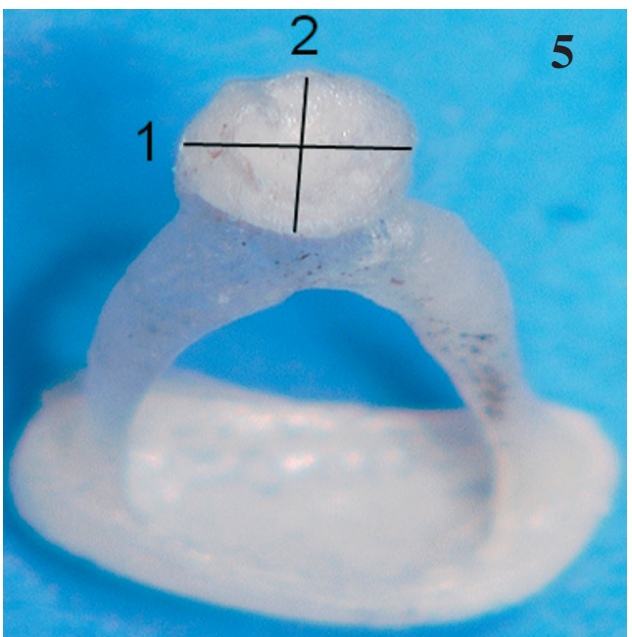

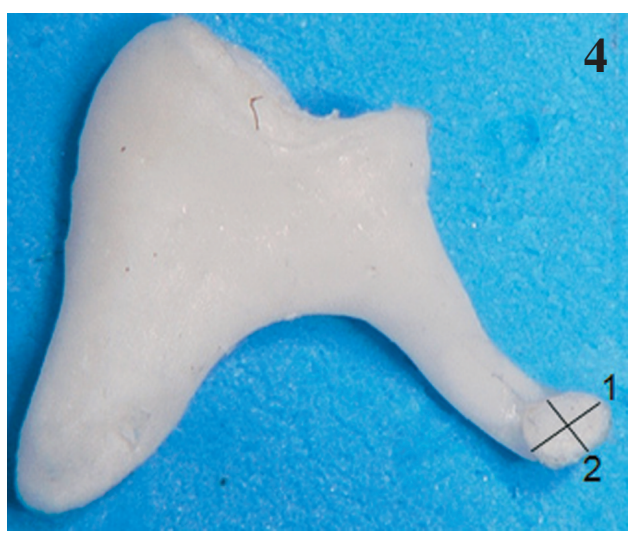

Fig. 5. Dimensions of the stapedial articular facet of the incus on the semimajor antero-posterior axis (1) and on the semiminor supero-inferior axis (2).

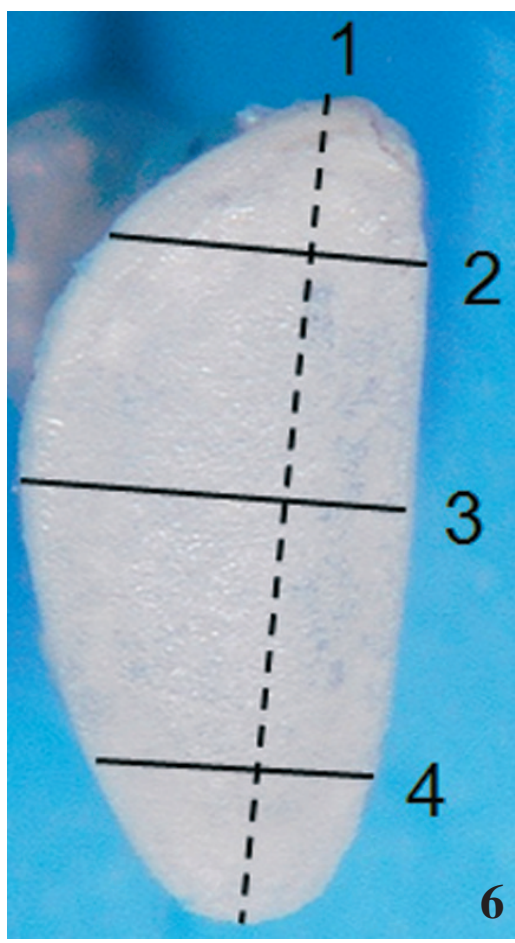

Fig. 7. Dimensions of the oval articular face of the stapes on a semimajor anteroposterior axis (1) and on three semiminor supero-inferior axes $(2,3,4)$. 
RAMIREZ, L. M. \& BALLESTEROS, L. E. Human oscicular chain articulations: Asymmetric sound transmission. Int. J. Morphol., 28(4):1059-1068, 2010.

Table I. Parametric measurements of the articular areas are given in $\mathrm{mm}^{2}$ calculated with the ellipse formula from 23 samples.

\begin{tabular}{|c|c|c|c|c|c|c|}
\hline & $\mathrm{T}$ & M-I & I-M & I-S & S-I & $\mathrm{S}-\mathrm{O}$ \\
\hline $\mathrm{n}$ & 23 & 23 & 23 & 23 & 23 & 23 \\
\hline average & 190.96 & 15.87 & 8.11 & 1.45 & 3.35 & 12.64 \\
\hline variance & 1098.87 & 7.22 & 2.97 & 0.12 & 0.40 & 0.99 \\
\hline SD & 33.15 & 2.69 & 1.72 & 0.35 & 0.63 & 0.99 \\
\hline
\end{tabular}

T: tympanum, M-I: area of incudomalleolar joint in the malleus, I-M: area of incudomalleolar joint in the incus, I-S: area of lenticular articulation in the incus, S-I: area of lenticular articulation in the stapes, S-O: area of articulation for the footplate of the stapes in the oval window.

Table II. Pearson correlations between the area of the tympanic membrane (T) and the articular areas of the oscicles (M-I, I-M, I-S, S-I, S-O).

\begin{tabular}{lllllll}
\hline & $\mathrm{T}$ & $\mathrm{M}-1$ & $\mathrm{I}-\mathrm{M}$ & $\mathrm{I}-\mathrm{S}$ & $\mathrm{S}-1$ & $\mathrm{~S}-0$ \\
\cline { 2 - 7 } $\mathrm{T}$ & 1,000 & & & \\
$\mathrm{M}-\mathrm{I}$ &,- 109 & 1,000 & & & \\
$\mathrm{I}-\mathrm{M}$ &,- 208 &, 080 & 1,000 & & \\
$\mathrm{I}-\mathrm{S}$ &, 416 &, 136 &, 214 & 1,000 & & \\
$\mathrm{~S}-1$ &, 222 &, 011 &, 489 &, 515 & 1,000 & \\
$\mathrm{~S}-0$ &, 210 & 0,32 &, 226 &, 034 &, 435 & 1,000 \\
\hline $\mathrm{n}=23$ & & \pm .413 & Critic value .05 two tales \\
& & \pm .5 .26 Critic value .01 two tales & & \\
\end{tabular}

T: tympanum, M-I: area of incudomalleolar joint in the malleus, I-M: area of incudomalleolar joint in the incus, I-S: area of lenticular articulation in the incus, S-I: area of lenticular articulation in the stapes, S-O: area of articulation for the footplate of the stapes in the oval window.

\section{Area Comparation}

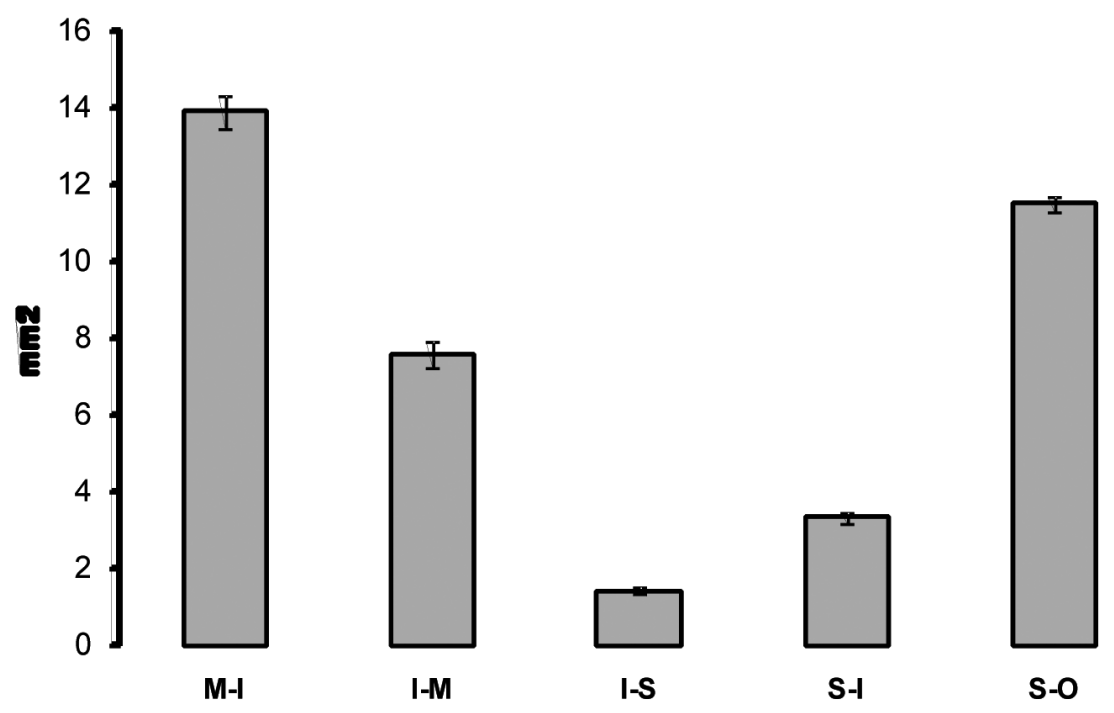

Fig. 8. Graphical representation of the articular areas $\left(\mathrm{mm}^{2}\right)$ of the oscicles from the incudomalleolar articulation to the oval window. M-I: area of incudomalleolar joint in the malleus, I-M: area of incudomalleolar joint in the incus, I-S: area of lenticular articulation in the incus, S-I: area of lenticular articulation in the stapes, S-O: area of articulation for the footplate of the stapes in the oval window. 


\section{Paired differences for areas of articulation}

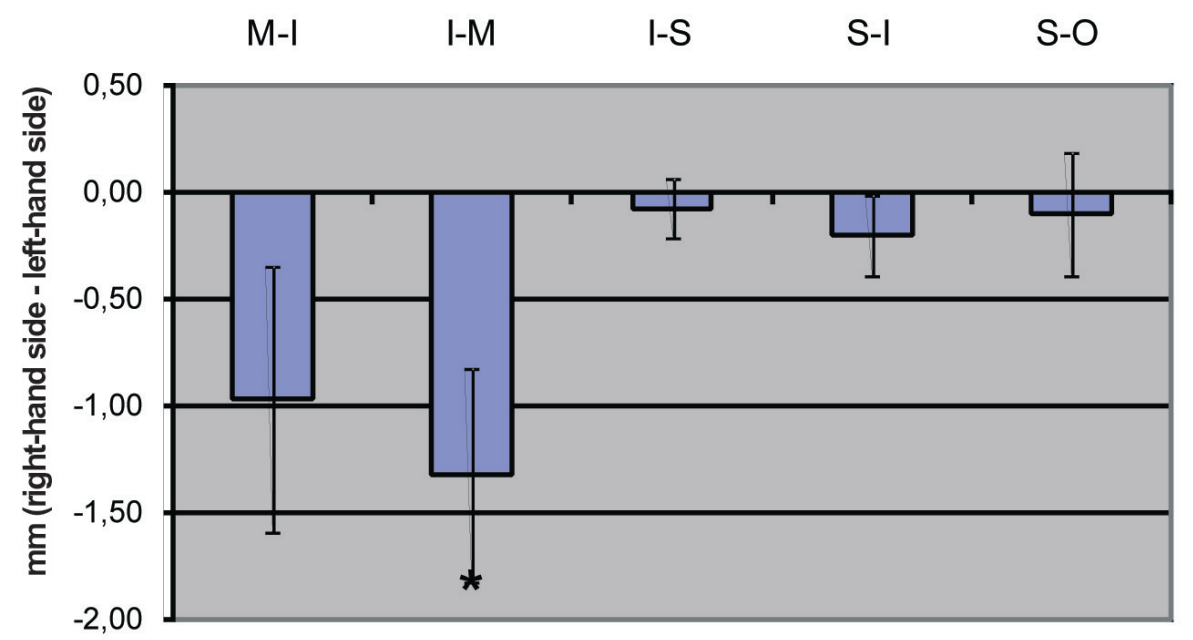

Fig. 9. Side relationship of paired samples for articular areas for the oscicles from the incudomalleolar joint to the oval window. M-I: area of incudomalleolar joint in the malleus, IM: area of incudomalleolar joint in the incus, I-S: area of lenticular articulation in the incus, SI: area of lenticular articulation in the stapes, S-O: area of articulation for the footplate of the stapes in the oval window.

An average measurement of $1.18 \mathrm{~mm}(0.11 \mathrm{SD})$ was obtained on the incus articular facet of the stapes (Fig. 5) on the major axis (antero-posterior) and $0.90 \mathrm{~mm}(0.11 \mathrm{SD})$ on the minor supero-inferior axis.

Three measurements were obtained on the articular (oval) face of the stapes on the minor (supero-inferior) axis and one on the major (antero-posterior) axis. The most posterior averages on the minor axis were $1.15 \mathrm{~mm}(0.14 \mathrm{SD}) 1.36$ $\mathrm{mm}(0.09 \mathrm{SD})$ intermediate and $1.32 \mathrm{~mm}(0.18 \mathrm{SD})$ for the most anterior. The measurement was $2.87 \mathrm{~mm}(0.09 \mathrm{SD})$ on the major axis.

Average (Table I) area was calculated in $\mathrm{mm}^{2}$ for the tympanic membrane, the oval window and each articular surface corresponding to the oscicles. They are represented in Figure 7 and the corresponding Pearson correlation is shown in Table II. Figure 8 shows the side correlation between paired samples.

\section{DISCUSSION}

It must be understood from the start that impedance is expressed in mass (small bones and tympanic membrane), toughness and elasticity at low frequencies, contrary to high frequencies where mass prevails. Peripheral mass, toughness, elastic resistance and frictional resistance are taken into account in the filtrate hypothesis when configuring the impedance of sound transmission through air; the effective volume of the middle ear, the toughness of the tympanum and the oscicles' degree of mobility are also related to elastic resistance. The conduction of high, medium and low frequencies is attenuated and filtered by the oscicles (air impedance) but also by the cochlea (liquid impedance) due to their frictional resistance, mainly at low frequencies. Bearing this in mind, the characteristics and configuration of the synovial articulations of the oscicles play a major role in conducting sound energy since they do not have the same effect at low frequencies as they have less friction in their rotational movements.

It must be considered that the tympanum's maximum displacement in response to an intense sound stimulus records a maximum nanometric deformation value $(1-4 \mathrm{~nm})$ which varies in relationship to frequency (Tonndorf \& Khanna). The oscicular chain may also transmit such subtle vibration of the tympanum by means of its diarthrosic articular facets through two articulations and three bones in the middle ear to the liquid environment of the internal ear having a relationship of areas and levers which are apparently safe for human sound frequencies $(18-15,000$ Hertz) (Heiland et al.; Whyte et al., 2008, Hato et al.).

Following the above, and from a morphological and morphometric perspective, it should be explained that the oscicular structures are irregular and unbalanced when the articular facet of each of these bones is taken as reference. Such characteristic produces a mechanical lever effect in 
sound transmission due to the moments of force produced by this morphology when they are articulated.

The relationship of oscicular articular areas did not show a strict lineal relationship from the tympanic membrane to the oval window in the present study regarding the transmission of sound energy. Starting with the tympanic membrane, an area of around $190.9 \mathrm{~mm}^{2}$ was obtained in this investigation (Table I). It should be mentioned that tympanic areas of around $55-65 \mathrm{~mm}^{2}$ have been reported in the literature (Guyton \& Hall); such measurements disagree with those found in the present investigation using the ellipse formula, being three times higher. However, it has also been stated that the difference in area between the tympanic membrane and the oval window is 17 times greater (Guyton \& Hall), meaning that when adjusted to our ellipse calculations for the area obtained in the oval window, which was about $11.5 \mathrm{~mm}^{2}$ (Table I), and then multiplied by 17, it approached that for the tympanic area with the ellipse formula $\left(190.9 \mathrm{~mm}^{2}\right)$. This is important in principle due to the fact that calculations may differ in tympanic area but are maintained in proportion to the areas of the articular surfaces being calculated (Table I and Fig. 7), thereby leading to reliable results.

The Pearson correlation matrix between the articular areas of the oscicles was expressed by a critical value $(0.05)$ for most articulations present in sound transmission for the middle ear's chain of bones. These results suggested a relationship of effective levers and areas in conducting sound in which the incus appeared to play an intermediate and critical role in such conduction. The power of the correlation- ship for the 23 samples fluctuated between 0.416 and $0.515(0.05$ critical value), seeming to be related to the articular closeness of the oscicular complex. In this range of power (from minor to major), these correlations were for the areas of the tympanum and the lenticular articulation of the incus, the articulation of the footplate of the stapes in the oval window, the area of incudomalleolar joint in the incus and the area of lenticular articulation in the stapes and the area of lenticular articulation in the incus with that corresponding to the stapes, the greatest power being shown for the latter (Fig. 9).

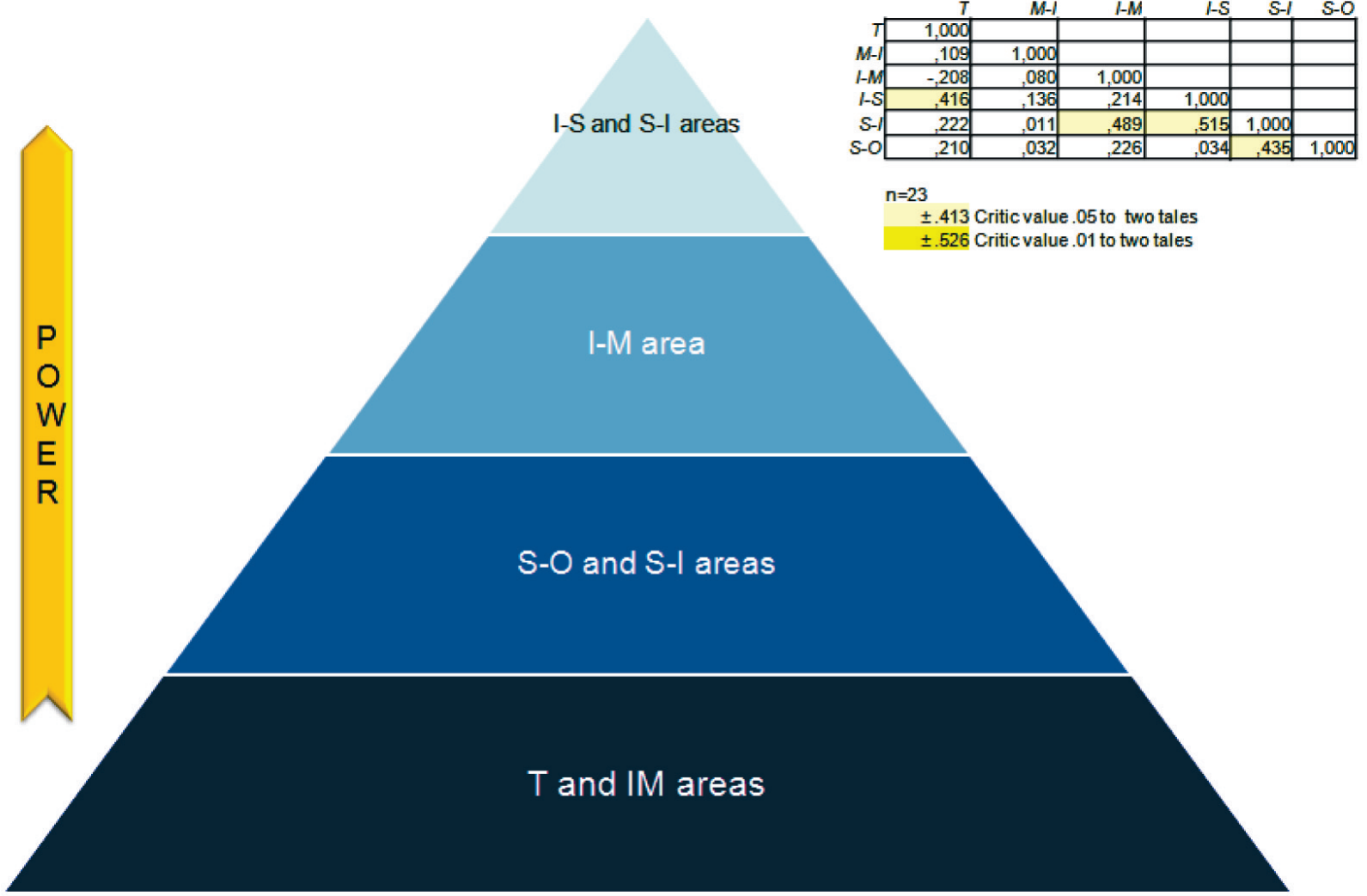

Fig.10. Important correlations between articular areas having .05 and .01 two-tailed test critical values, showing lesser to greater statistical power (yellow arrow). Table 2 (shown in the upper right-hand corner) relates the origin of the pyramid regarding its statistical power. I-S and S-I areas: Incus and stapedial incudo-stapedial areas, I-M area: Incudo-malleolar incus area, S-O and S-I areas: area of articulation for the footplate of the stapes in the oval window and area of lenticular articulation in the stapes, T and I-M areas: tympanic and incudo-malleolar incus areas. 
The way in which the articular areas become reduced (from greater to smaller) from the facet of the malleus to the facet of the lenticular articulation, to be amplified later on from an inverse relationship of areas (from smaller to greater) from the same lenticular articulation in the head of the stapes to the oval window (Fig. 7), suggests a complex relationship in transmitting energy through these small bones mobility and seems to be related to the type of lever, the size of articular facets and the dimensions of each bone involved in these transmission mechanics. However, it is worth explaining that even though such asymmetric relationships are opposed, the oscicles appear to perform a double function: amplificationreduction or reduction-amplification.

Which of these two possibilities really occurs must thus be defined. Interesting correlations were made which might explain the foregoing and which have been supported by additional measurements of the oscicular chain (not completely shown in these results but partially revealed in Table III). One of these occurred between the size of the incudo-malleolar articular face and the amount of separation of the short and long apophysis of the incus $(0.416$, having $0.05=0.413$ critical value) seeming to have modulator ability regarding these mechanics, thus implying that a greater separation of both apophysis of the incus (higher lever's arm) would lead to a greater size in the area of the articular face and an effect on sound transmission capacity based on the information obtained from the average of the areas explained above. This

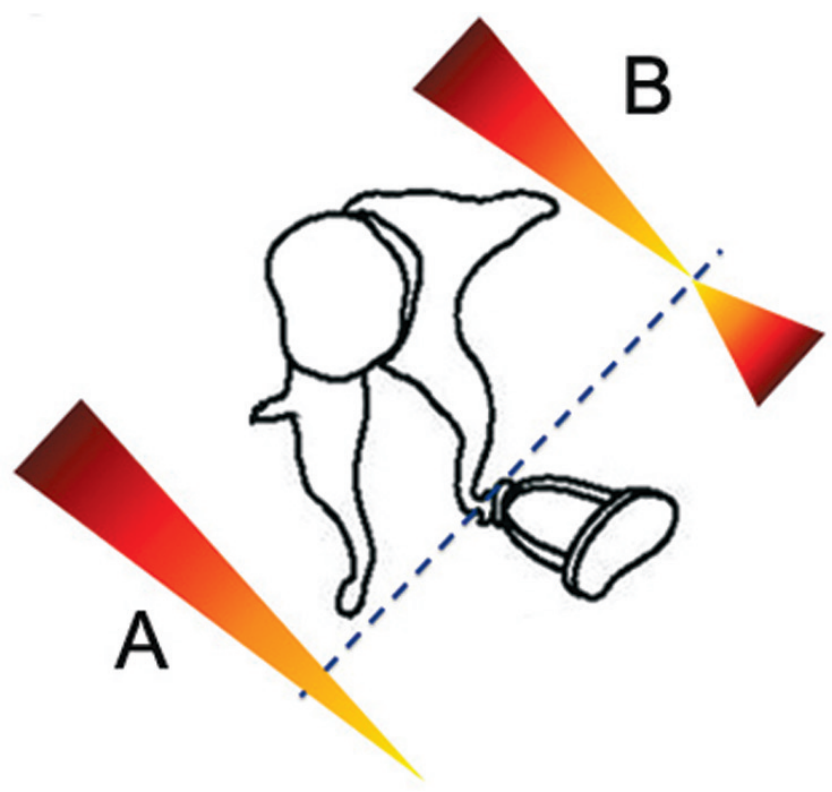

Fig. 11. Two representations of sound conduction regarding air impedance in the middle ear. The lower funnel (A) corresponds to Nakajima et al.'s model and the double upper funnel (B) corresponds to our results, showing the transmission of filtered energy in the incudostapedial joint (dotted line).
Table III. Correlations between articular areas and the amount of separation of short and long apophysis of the incus and the height of the stapes. I-M: area of incudomalleolar joint in the incus, S-I: area of lenticular articulation in the stapes.

\begin{tabular}{llc}
\hline VARIABLES & $\mathrm{I}-\mathrm{M}$ & $\mathrm{S}-1$ \\
\hline $\begin{array}{l}\text { Long-Short } \\
\text { Platine Height }\end{array}$ &, 416 & -465 \\
\hline $\mathrm{n}=23$ & & \pm .413 : Critical value .05 \\
\hline
\end{tabular}

information suggested that sound transmission thus seemed to count with synovial articulations that range the acoustic transmission from greater to lesser (reducing), at the beginning (from tympanic membrane to incudostapedial joint).

Another inverted co-relationship (Table III), which could complement that explained above regarding the rest of the transmission from incudostapedial joint, was presented between the size of the incudostapedial joint of the stapes and their height $(-0.465$, having $0.05=0.413$ critical value $)$. A stapes in which its cruras are not high would present a broader articular facet. Such inverted relationship to that explained in the previous paragraph with the incus and the tympanic membrane thus shows that an increase in the size of articular facet somehow compensates for a shorter lever arm. This may be understood as having a more medial amplifying effect on the oscicles in this segment. These two articulations (incudomalleolar and incudostapedial), from their articular areas, seem to be located at both sides of the inversion of the mechanics reducing and amplifying sound transmission, suggesting that the relationship of areas and sound transmission from the tympanic membrane to the oval window is first a reducing one and then an amplifying one. This disagrees with Nakajima et al.'s model (Fig. 9).

Nakajima et al. have stated that the oscicular chain does not use levers to magnify force per surface unit from the tympanic membrane to the annular ligament of the oval window and, on the contrary, becomes reduced, cushioning such energy with the elasticity inherent in all articulations involved. This goes against the belief that the oscicles' scheme of levers amplifies an initial mechanical effect on the tympanic membrane without increasing the amount of displacement of the stapes but increases the pushing force 1.3 times (Guyton $\&$ Hall). Our results partly agree with this as the arms of the lever originating in these bones' greater length becoming reduced tends to interfere with a relationship of lineal and effective areas in the incus; however, they then become increased in the articular face of the stapes in their lineal relationship from the incudostapedial joint to the area of the annular articulation of the oval window.

Such asymmetric mechanical relationship may have an explanation in the mechanism of auditory protection and 
discrimination in which the tensor tympani and stapedial muscles (through centrifugal reflex) may modulate vibratory ability on being inserted, respectively, into the handle of the malleus and the neck of the stapes (Ramirez et al., 1989). The entry of sound energy may thus be directly modulated at the start towards the tympanum-malleus by the tensor tympani muscle. Additionally, sound conduction can also be modulated in the incudostapedial joint by stapedial muscle. This being the breaking-point for this group of oscicular levers which is coincidentally located in the same place as that for the relationship of articular areas is inverted. Incudostapedial joint thus seems to show an important incus in the connection and discretionary mechanics of such sound transmission, playing the role of "fulcrum" with the stapedial muscle as motor regulator (low frequency protector and discriminator) of this transmission.

Even though the limits of this study must be recognised, some deductions may be made from the data obtained and correlated, thereby leading to more questions being posed. One such would concern the mechanics of sound energy transmission from the data so obtained. It was thus found that there was no lineal relationship of the areas of the oscicles in the samples used here. It must be kept in mind that other species different to mammals (having their unique characteristics regarding the number of small bones, tympanic membrane and cochlear discrimination mechanisms), such as reptiles, fish, amphibians and birds, cannot perceive high sound frequencies. Some of them have just a small bone bound to the tympanic membrane, having a direct relationship between its vibration and the vibration of the stapes footplate (columela) (Borg \& Counter). This type of oscicular mechanics has an effect on the type of movement of the stapes footplate (pivot) different to that of mammals, including humans, at different frequencies and which are almost certainly modulated by the incus and its incudostapedial joint (Heiland et al.; Hato et al.). The middle ear's evolution in mammals has demanded efficient mechanisms battling with noises having different frequencies and needing to be discriminated as a mechanism in which survival has depended on the ability to localise the origin of noise. It is thus thought that the forms, areas and levers in the middle ear, added to the presence of tensor muscles, can explain topics related to mammals' exclusive evolution (Puria $\&$ Steele, 2009).

No side differences were found in most paired variables measured in this study; however, the area of incudomalleolar joint in the incus did show difference (Fig. 9). It is worth mentioning that no record was found of investigations in which the samples have been paired and studied from such viewpoint.

In conclusion, a complex relationship of levers could be observed in which irregular forms of the oscicles and their articular facets generated an asymmetric relationship, suggesting an initial amplifying sound transmission function and a final reducer destination for such transmission from the incudostapedial joint.

\section{ACKNOWLEDGEMENTS}

We would like to thank the Forensic Medicine Institute (Instituto de Medicina Legal) for its much appreciated donation of samples.

RAMIREZ, L. M. \& BALLESTEROS, L. E. Articulaciones de la cadena oscicular humana: Transmisión asimétrica del sonido. Int. J. Morphol., 28(4):1059-1068, 2010.

RESUMEN: El mecanismo para conducir la energía acústica a través de oscículos del oído medio es todavía un tema controvertido y lo seguirá siendo hasta alcanzar un consenso con respecto a si se amplía o reduce la transmisión del sonido. Fueron estudiados 22 pares de bloques de hueso temporal izquierdo y uno derecho hmanos. Mediciones digitales fueron tomadas de la membrana timpánica, articulaciones osciculares y de la ventana oval, siendo estos resultados correlacionados. Una relación de las áreas no-lineal se encontró entre las estructuras, lo que sugiere una relación de transmisión de sonido que incluye tanto la reducción de sonido y amplificación. Una compleja relación de las palancas se pudo observar originando una relación oscicular y articular asimétrica, lo que sugiere una función de amplificación de la transmisión del sonido inicial y también un destino reductor final para su conducción.

PALABRAS CLAVE: Malleus; Incus; Estapedio; Tímpano; Articulación incudomalleolar; Articulación incudostapedial; Ventana oval; Músculo estapedio.

\section{REFERENCES}

Anson, B. J. \& Donaldson, J. A. Surgical anatomy of the temporal bone. $3^{\text {rd }}$ ed. Philadelphia, W. B. Saunders, 1991.
Asai, M.; Heiland, K. E.; Huber, A. M. \& Goode, R. L. Evaluation of a cement incus replacement prosthesis in a temporal bone model. Acta Otolaryngol., 119:573-6, 1999. 
Arensburg, B. \& Nathan, H. Observations on a notch in the short (superior or posterior) process of the incus. Acta Anat., 78:84-90, 1971.

Aycan, K.; Unur, E.; Bozkır, M. G.; et al. Anatomical study of malleus. J. Health Sciences, 1:152-58, 1990.

Borg, E. \& Counter, S. A. The middle-ear muscles. Sci. Am., 261:74-80, 1989.

Coleman, M. N. \& Colbert, M. W. Correlations between auditory structures and hearing sensitivity in non-human primates. J. Morphol., 271:511-32, 2010.

Crevecoeur, I. New discovery of an Upper Paleolithic auditory ossicle: the right malleus of Nazlet Khater 2. J. Hum. Evol., 52:341-5, 2007.

Guyton, A. C. \& Hall, J. E. Tratado de Fisiología Médica. $11^{\circ}$ Ed. Barcelona, Elsevier S.A., 2007.

Hato, N.; Stenfelt, S. \& Goode, R. L. Three-dimensional stapes footplate motion in human temporal bones. Audiol. Neurootol., 8:140-52, 2003.

Heiland, K. E.; Goode, R. L.; Asai, M. \& Huber, A. M. A human temporal bone study of stapes footplate movement. Am. J. Otol., 20:81-6, 1999.

Kassem, F.; Ophir, D.; Bernheim, J. \& Berger, G. Morphology of the human tympanic membrane annulus. Otolaryngol. Head Neck Surg., 142:682-87, 2010.

Murugasu, E.; Puria, S. \& Roberson, J. B. Jr. Malleus-tofootplate versus malleus-to-stapes-head ossicular reconstruction prostheses: temporal bone pressure gain measurements and clinical audiological data. Otol. Neurotol., 26:572-82, 2005.

Mills, R. P. Ossicular geometry and the choice of technique for ossiculoplasty. Clin. Otolaryngol. Allied. Sci., 16:4769, 1991.

Nakajima, H. H.; Ravicz, M. E.; Merchant, S. N.; Peake, W. T. \& Rosowski, J. J. Experimental ossicular fixations and the middle ear's response to sound: evidence for a flexible ossicular chain. Hear Res., 204:60-77, 2005.

Nishihara, S. \& Goode, R. L. Experimental study of the acoustic properties of incus replacement prostheses in a human temporal bone model. Am. J. Otol., 15:485-94, 1994.

Olsewski, J. Structure of the middle ear in infants. Otolaryngol. Pol., 43:278-83, 1989.
Olsewski, J. The morphometry of the ear ossicles in humans during development. Anat. Anz., 171:187-91, 1990.

Proop, D.; Hawke, M.; Berger, G. \& MacKay, A. The anterior process of the malleus. J. Otolaryngol., 6:257-62, 1984.

Puria, S. \& Steele, C. Tympanic-membrane and malleus-incuscomplex co-adaptations for high-frequency hearing in mammals. Hear Res., 1:8, 2009.

Ramirez, L. M.; Ballesteros, L. E. \& Sandoval, G. P. Tensor veli palatini and tensor tympani: anatomical, functional and symptomatically link. Act. Esp. Otorrinolaringol., 61:2633,2010 .

Sarrat, R.; Guzman, G. \& Tores, A. Morphological variations of human ossicular tympani. Acta Anat., 131:146-9, 1988.

Sun, Q.; Gan, R. Z.; Chang, K. H. \& Dormer, K. J. Computerintegrated finite element modeling of human middle ear. Biomech. Model Mechanobiol., 1:109-22, 2002.

Tonndorf, J. \& Khanna, S. M. Tympanic-membrane vibrations in human cadaver ears studied by time-averaged holography. J. Acoust. Soc. Am., 52:1221-33, 1972.

Wassif, K. The anterior process and the ossification of the malleus in mammals. Science, 113:486-7, 1951.

Wengen, D. F.; Nishihara, S.; Kurokawa, H. \& Goode, R. L. Measurements of the stapes superstructure. Ann. Otol. Rhinol. Laryngol., 104:311-6, 1995.

Whyte, J.; Cisneros, A. I.; Urieta, J. J.; Yus, C.; Gañet, J.; Torres, A. \& Sarrat, R. Ontogenic peculiarities of the human tympanic ossicular chain. Acta Otorrinolaringol. Esp., 54:110, 2003.

Whyte, J.; Cisneros, A.; Yus, C.; Obón, J.; Whyte, A.; Serrano, P.; Pérez-Castejón, C. \& Vera, A. Development of the dynamic structure (force lines) of the middle ear ossicles in human foetuses. Histol. Histopathol., 23:1049-60, 2008.

Correspondence to:

Dr. Luis Miguel Ramirez.

Calle $45 \mathrm{~N}^{\circ}$ 33-33. Apto 702b.

Bucaramanga

COLOMBIA

\section{Cell Phone: 316-7536838}

Email: Imra3@yahoo.com

Received : 10-07-2010

Accepted : 26-09-2010 